\title{
Seguimiento de anfibios en espacios protegidos del País Vasco y Navarra. I. Parques Naturales de Izki y Valderejo (Álava).
}

\section{Monitoring of amphibians in protected spaces in the Basque Country and Navarre. I. Natural Parks of Izki and Valderejo (Alava).}

Alberto Gosá*, Ion Garin-Barrio, Carlos Cabido, Aitor Laza-Martínez, Xabier Rubio

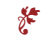

\section{Resumen}

Entre 2010 y 2017 se censaron las poblaciones de anfibios en una serie de humedales de los parques naturales de Izki y Valderejo (Álava) durante la estación reproductora y el periodo de desarrollo larvario, con el objetivo de analizar su tendencia. Se constata el declive de especies típicamente mediterráneas en ambos parques, en especial de Discoglossus galganoi Capula, Nascetti, Lanza, Crespo \& Bullini, 1985 y Pelodytes punctatus (Daudin, 1802), que no han vuelto a ser localizados en Valderejo desde la década de 1990, al menos en los humedales donde estaban presentes en esa época y que fueron muestreados posteriormente, así como de $D$. galganoi en Izki. Las restantes especies parecen encontrarse estabilizadas en ambos parques, siendo de destacar el crecimiento de la población de Triturus marmoratus (Latreille, 1800) en Valderejo y su reducción en Izki.

Palabras clave: conservación, muestreo de poblaciones, declive de anfibios, herpetofauna, Parque Natural de Izki, Parque Natural de Valderejo, especies mediterráneas, Álava.

\section{Abstract}

Between 2010 and 2017, during the breeding season and the period of larval development, we conducted censuses of amphibian populations in a representative sample of ponds, in the natural parks of Izki and Valderejo (province of Alava, Basque Country).

\footnotetext{
1 Sociedad de Ciencias Aranzadi. Departamento de Herpetología.

Paseo de Zorroaga 11, 20004 Donostia-San Sebastián (Spain)

*Correspondencia: agosa@aranzadi.eus
} 
A decline in typically Mediterranean species has been recorded in both parks. Specifically, since the 1990s, Discoglossus galganoi Capula, Nascetti, Lanza, Crespo \& Bullini, 1985 and Pelodytes punctatus (Daudin, 1802) have not been found in Valderejo, at least in the wetlands where they were present during that period and were subsequently detected. The status of $D$. galganoi in Izki is similar. The situation of the remaining species seems to be stable in both parks, whereas the population of Triturus marmoratus (Latreille, 1800) has grown in Valderejo, but decreased in Izki.

Key words: conservation, population census, decline in amphibians, herpetofauna, Izki Natural Park, Valderejo Natural Park, Mediterranean species, Alava.

\section{Laburpena}

2010-2017 urteen artean Izki eta Valderejoko parke naturaletako (Araba) hezegune batzuetan anfibio populazioak zenbatu egin ziren ugaltze garaian eta larben garapen fasean, beraien joera jakiteko. Bada, bi parkeetan ohiko espezie mediterraneoen gainbehera egiaztatu da, batez ere Discoglossus galganoi Capula, Nascetti, Lanza, Crespo \& Bullini, 1985 eta Pelodytes punctatus (Daudin, 1802), biak ere 1990eko hamarkadatik ez baitira berriro Valderejon aurkitu, behintzat garai hartan topatu ziren hezeguneetan, gerora laginduak izan arren. Berdin gertatu da D.galganoi-ren kasuan Izkin. Gainontzeko espezieak bi parkeetan egonkor mantentzen direla dirudi, eta azpimarratzekoa da Triturus marmoratus (Latreille, 1800) populazioaren gorakada Valderejon eta bere gainbehera Izkin.

Gako hitzak: kontserbazioa, populazioen laginketa, herpetofauna, Izki Parke Naturala, Valderejo Parke Naturala, Araba.

$$
\text { r. } x
$$

\section{Introducción}

El seguimiento de los anfibios en series temporales largas es un método comúnmente utilizado para conocer la evolución de las poblaciones, que puede servir para definir su tendencia e incluso poner en evidencia algunas de las posibles causas que la determinan. Las afecciones producidas por una era de cambio climático, contaminación creciente y recurrente aparición de especies invasoras como la que estamos viviendo hacen, aún más si cabe, aconsejable la realización de censos periódicos de las poblaciones siguiendo metodologías estandarizadas (Heyer et al., 1994). Dichas metodologías facilitan el análisis comparativo, tanto en coordenadas temporales como espaciales. Si la comparación de resultados interanuales (coordenadas temporales) para las poblaciones de un mismo enclave no ofrece dificultad alguna de comprensión, la de enclaves diferentes (coordenadas espaciales) sí puede presentar situaciones que complican la interpretación. Conviene comparar abundancias interanuales en los mis- 
mos enclaves para interpretar la evolución de las tendencias poblacionales en las mejores condiciones, pero esto no siempre es posible por diversas causas logísticas, que impiden visitar los mismos humedales cuando ha trascurrido un amplio número de años. En tales casos, habrá que ceñirse a los humedales repetidamente muestreados e intentar que el número y su representatividad como biotopos reproductores de los anfibios sean los más adecuados. Por otra parte, la preferencia de los anfibios por las masas acuáticas de pequeñas dimensiones y la relativa homogeneidad estructural que éstas presentan en el bosque templado del norte ibérico, facilitan el muestreo y pueden llegar a facilitar las comparaciones, aportando resultados útiles en los programas de gestión. Sin embargo, no todas las especies van a poder beneficiarse de esta circunstancia, especialmente aquéllas adaptadas a medios efímeros e inestables que pueden verse altamente modificados o incluso desaparecer, siendo imposible contar con ellos en los subsiguientes muestreos, lo que al mismo tiempo puede introducir factores de riesgo en la evaluación e interpretación del estado de sus poblaciones.

La selección de lugares apropiados para la puesta en funcionamiento de programas de seguimiento depende de diversos factores. Uno que puede ser decisivo es su importancia, en términos medioambientales y socioeconómicos, por lo que los espacios naturales protegidos suelen ser, comprensiblemente, objeto prioritario de atención (Santos et al., 1998) en los programas europeos (p.e. la Directiva de Hábitats), los cuales exigen a las administraciones el seguimiento periódico de especies amenazadas. Algunas proyecciones de cambios climáticos futuros predicen pérdidas de biodiversidad (número de especies de vertebrados y plantas) en espacios protegidos, entre los que se consideran los parques naturales, que incluso serían superiores en los espacios de la Red Natura 2000 (zonas especiales de conservación), donde podrían afectar en 2080 al 63 \% de las especies (Araújo et al., 2011). Para esa fecha ciertos escenarios predicen mayores pérdidas de idoneidad climática en especies de anfibios que ganancias (especialmente en espacios de la Red Natura 2000), mientras que otros predicen más ganancias que pérdidas (en parques naturales) para esta fauna.

Con los datos disponibles, se delimitaron en su día para el País Vasco siete zonas de interés herpetológico, coincidentes en su mayoría con espacios protegidos (Mateo, 2002). Recientemente, se ha sugerido preliminarmente que los espacios protegidos en el País Vasco podrían conformar una red con suficiente entidad para asegurar la conservación de esta fauna (Crespo Diaz \& Tamayo, 2007). Se dispone de censos en algún lugar de importancia comunitaria, como el de Salburua (Ekos, 2006; Pagoa, 2010). Sin embargo, la presencia generalizada de los anfibios aun en lugares históricamente modificados por el hombre, introduce elementos de discusión que sugieren que la gestión también puede llevarse a cabo en este tipo de ambientes. En el País Vasco, esta circunstancia vendría constatada por la existencia de poblaciones amenazadas en áreas no protegidas (Garin-Barrio et al., 2007; Olano et al., 2007; Etxezarreta et al., 2007; Crespo Diaz et al., 2007; Crespo Diaz, 2008), con frecuencia sustentadas 
por la permanencia en ellas de humedales cuyo origen viene a ser mayoritariamente artificial, con el objetivo de servir a la gestión ganadera y agrícola. En áreas a gran escala y en faunas con mayor movilidad que la de los anfibios, como los mamíferos terrestres, la conectividad entre las áreas protegidas nacionales y trasnacionales resulta clave para la conservación (Santini et al., 2015).

Desde la década de 1990, diferentes equipos de trabajo han realizado censos de las poblaciones de anfibios en dos parques naturales de montaña del País Vasco: Izki y Valderejo (Álava) (Fig. 1). El Parque Natural de Izki ocupa el extremo oriental del cordal de sierras que atraviesa la provincia de Álava de oeste a este en su zona centro-sur. Izki está dominado por masas de Quercus pyrenaica Willd. situadas a una altitud media de 800 msnm. El Parque Natural de Valderejo, ubicado en la zona de Valdegovía, limita con la provincia de Burgos a la altura de la zona más occidental de Álava, y cuenta con una amplia representación de masas forestales de Pinus sylvestris L. y Fagus sylvatica L. combinadas con pastizales rasos, a una media altitudinal de en torno a los 1000 msnm. El primer censo de anfibios en estos parques fue realizado en 1999-2000 (Izki) y 1995 (Valderejo) (Onrubia et al., 1996; Onrubia et al., 2007). Pocos años después se repitió el de Valderejo (CRN, 1999), y hasta la década de 2010 no se han

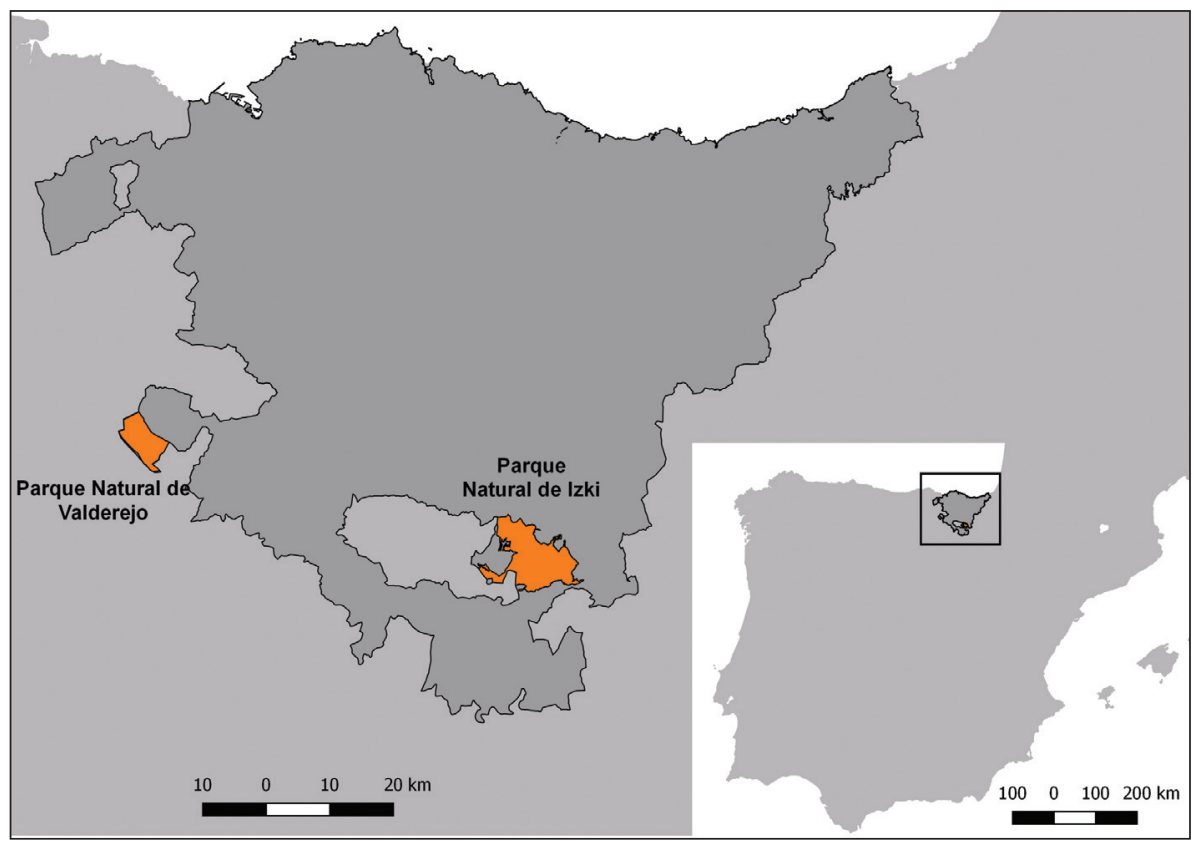

Fig 1.- Ubicación geográfica de los parques naturales de Valderejo e Izki (País Vasco).

Fig 1.- Geographic location of the natural parks of Valderejo and Izki (Basque Country). 
vuelto a muestrear (Gosá \& Iraola, 2010a y b; Gosá, 2011; Gosá \& Garin-Barrio, 2017; Gosá et al., 2017a; Gosá et al., 2017b).

Con el objetivo de actualizar la situación de las poblaciones de anfibios en los Parques Naturales de Izki y Valderejo, y de obtener una idea de su posible tendencia poblacional, se analizaron los resultados de los censos obtenidos entre 2010 y 2017 y se compararon con los realizados por otros equipos en décadas precedentes.

\section{Material y métodos}

El seguimiento se realizó en tres años en ambos parques: 2010, 2011 y 2017, mediante el censo, separado para adultos y larvas, de poblaciones en charcas, encharcamientos y cursos de montaña durante la estación reproductora y el periodo de desarrollo larvario. Se aplicó una metodología homogénea en los dos parques y en los diferentes años. Las charcas se muestrearon con salabre, desde las orillas o en su interior, en función de sus características estructurales. Unas fueron muestreadas en la totalidad de su superficie y otras, de mayores dimensiones o más complejas, en zonas seleccionadas por presentar las mejores cualidades para la presencia de anfibios y unas dimensiones asimilables a las de las charcas menores. Los arroyos fueron censados siguiendo transectos establecidos o en pozas donde se concentraban las poblaciones. En el Parque Natural de Valderejo se muestrearon en los tres años de estudio entre 25 y 27 humedales, obteniéndose censos en al menos dos años para 15 de ellos que también habían sido muestreados en la década de 1990, y situados a una altitud comprendida entre 843 y 1041 msnm. En el Parque Natural de Izki se muestrearon entre 10 y 15 humedales, coincidiendo 12 de ellos (663-760 msnm) en al menos dos de los tres años. Seis de estos humedales habían sido censados en la década de 1990.

En este estudio sólo se aportan los datos de esos 15 humedales de Valderejo y 12 de Izki muestreados de forma repetida (Tabla 1). En Valderejo fueron censados entre una y cuatro veces, y en Izki entre una y seis por año, lo que permitió realizar estimaciones de abundancia máxima, expresadas como densidad relativa para 20 minutos de muestreo. Los muestreos fueron diurnos, y sólo excepcionalmente se llevaron a cabo también por la noche. Los criterios utilizados en la selección de humedales tuvieron como objetivo alcanzar un tamaño de muestra suficiente para establecer comparaciones estadísticas, habiéndose perseguido, en la medida que permitía la logística aplicada en este último proyecto de seguimiento (2017), la coincidencia con los inventarios precedentes, incluidos los de la década de 1990. Las circunstancias que impulsaron la realización de los censos, la disponibilidad de listados de humedales en los parques y la logística aplicada a la selección de los mismos no permitieron la realización de los muestreos en la totalidad de los humedales que habían sido censados en la década 


\begin{tabular}{|c|c|c|c|}
\hline \multirow{2}{*}{ Humedal } & \multicolumn{2}{|c|}{ P.N. VALDEREJO } & \multirow{2}{*}{ Tipo } \\
\hline & $x$ & Y & \\
\hline Ozalla & 479111 & 4749754 & Charca permanente \\
\hline Purón & 478870 & 4750020 & Charca permanente \\
\hline Santa Gadea I & 483180 & 4745096 & Charco estacional \\
\hline Solinde I & 483093 & 4744441 & Charca permanente \\
\hline Solinde II & 482999 & 4744479 & Charca permanente \\
\hline Santa Ana & 481722 & 4747081 & Charca permanente \\
\hline Lalastra & 481404 & 4747191 & Charca permanente \\
\hline La Choza & 482694 & 4745846 & Charca temporal \\
\hline Polledo & 481715 & 4745537 & Charco estacional \\
\hline El Cubo & 479920 & 4746196 & Charca estacional \\
\hline Fuente Sus & 483134 & 4746371 & Charca permanente \\
\hline La Isa & 481747 & 4747427 & Charca estacional \\
\hline Andera & 480190 & 4746805 & Charca temporal \\
\hline Alto Llomo & 480743 & 4747731 & Charca permanente \\
\hline Cruz de san Miguel I & 481196 & 4746122 & Charco estacional \\
\hline \multirow{2}{*}{ Humedal } & \multicolumn{2}{|c|}{ P.N. IZKI } & Ting \\
\hline & $X$ & $\mathrm{Y}$ & 1100 \\
\hline Telecocharra II & 544525 & 4731826 & Charca permanente \\
\hline Dehesa Maeztu II & 544786 & 4730632 & Charca temporal \\
\hline Dehesa Maeztu III & 544733 & 4730556 & Charca temporal \\
\hline Fuente Honda & 541853 & 4724430 & Charca temporal \\
\hline Raso Espinos & 540397 & 4724166 & Charca temporal \\
\hline Peña el Acebal & 540808 & 4727803 & Charca permanente \\
\hline Galbaniturri & 539383 & 4727306 & Arroyo permanente \\
\hline Marizurieta & 539164 & 4726629 & Charca temporal \\
\hline Dehesa I & 542829 & 4723835 & Charca temporal \\
\hline Dehesa II & 542878 & 4723961 & Charco estacional \\
\hline El Mineral & 539615 & 4727389 & Arroyo permanente \\
\hline Galbaniturri II & 539419 & 4727276 & Trampal \\
\hline
\end{tabular}

Tabla 1.- Coordenadas geográficas (huso 30T) de los humedales censados en, al menos, dos de los tres años de seguimiento (datum WGS84).

Table 1.- Coordinates (zone 30T) of wetlands during at least two of the three years of monitoring (datum WGS84). 
de 1990, pero sí la repetición de los mismos en un número suficiente para establecer comparaciones estadísticas entre las dos épocas de estudio, al menos para las especies con presencia más asidua en los humedales.

\section{Resultados}

\section{Composición de las comunidades}

En el último decenio la comunidad anfibia de Valderejo ha fluctuado entre nueve (2010) y ocho $(2011,2017)$ especies, y la de Izki entre diez (2010), nueve (2011) y ocho (2017). La única especie no detectada por este estudio en Valderejo en 2017, con respecto a los comienzos de la década, ha sido Epidalea calamita (Laurenti, 1768). Por su parte, en Izki no se ha observado Discoglossus galganoi Capula, Nascetti, Lanza, Crespo \& Bullini, 1985 ni E. calamita en el último muestreo, habiéndose registrado, por el contrario, algún ejemplar de Salamandra salamandra (Linnaeus, 1758) en un humedal que no formaba parte de las zonas húmedas censadas y que, por tanto, no ha sido contabilizado. Otras especies compartidas en 2017 en ambos parques fueron Lissotriton helveticus (Razoumowsky, 1789), Triturus marmoratus (Latreille, 1800), Alytes obstetricans (Laurenti, 1768), Bufo spinosus Daudin, 1803, Hyla molleri Bedriaga, 1889 y Pelophylax perezi (López Seoane, 1885). Ichthyosaura alpestris (Laurenti, 1768) sólo estuvo presente en Valderejo, y Rana dalmatina Bonaparte, 1840 y Rana temporaria Linnaeus, 1758, en Izki, si bien esta última, que forma densos agregados de larvas en los humedales, aun habiendo sido localizada en algunos humedales de Valderejo, no fue censada por la dificultad de conteo y por haber coincidido los muestreos con una época poco representativa para su estimación poblacional.

\section{Censo poblacional en Valderejo}

Las diferencias en las abundancias máximas medias en la década de 2010 fueron altamente significativas entre especies en las poblaciones de adultos (Kruskal-Wallis: $\mathrm{H}=32$,39; $\mathrm{P}<0,001$ ). Por el contrario, en las larvarias las abundancias sólo fueron significativamente superiores en $A$. obstetricans frente a I. alpestris (Mann-Whitney: $U=16 ; P=0,022)$ y $B$. spinosus $(U=17,5 ; P=0,029)$. En las poblaciones de adultos, $L$. helveticus y $P$. perezi presentaron los valores más altos y, en las larvarias, lo fueron H. molleri, T. marmoratus y A. obstetricans (Tabla 2). Las abundancias de las poblaciones a lo largo de la década, tanto de adultos como de larvas, no variaron significativamente, a excepción de las de los adultos de $T$. marmoratus $\left(F_{2,27}=3,92\right.$; $P=0,031)$ y $P$. perezi $(H=4,71 ; P=0,012)$, que crecieron.

En la década de 1990 las abundancias de adultos presentaron diferencias significativas (datos elaborados de Onrubia et al., 1996 y CRN, 1999) en la población de L. helve- 


\begin{tabular}{|c|c|c|c|c|}
\hline Estadio & Especie & $\begin{array}{c}\text { Media } \\
\text { (error estándar) }\end{array}$ & $\mathrm{n}$ & Rango \\
\hline \multirow{9}{*}{$\begin{array}{l}\text { Adultos } \\
\text { (década } \\
\text { 1990) }\end{array}$} & L. helveticus & $14,79(3,30)$ & 24 & $0-56$ \\
\hline & T. marmoratus & $0,91(0,26)$ & 24 & $0-5$ \\
\hline & I. alpestris & $1,16(1,16)$ & 6 & $0-7$ \\
\hline & A. obstetricans & $1,50(0,73)$ & 14 & $0-10$ \\
\hline & D. galganoi & 1 & 2 & $0-1$ \\
\hline & B. spinosus & $2,25(1,06)$ & 12 & $0-12$ \\
\hline & E. calamita & $1,25(0,94)$ & 4 & $0-4$ \\
\hline & H. molleri & $3,66(1,48)$ & 12 & $0-15$ \\
\hline & P. perezi & $15,77(6,48)$ & 18 & $0-100$ \\
\hline \multirow{11}{*}{$\begin{array}{l}\text { Larvas } \\
\text { (década } \\
\text { 1990) }\end{array}$} & S. salamandra & $0,06(0,06)$ & 16 & $0-1$ \\
\hline & L. helveticus & $4,09(2,52)$ & 21 & $0-53$ \\
\hline & T. marmoratus & $2,76(2,37)$ & 21 & $0-50$ \\
\hline & I. alpestris & $0,25(0,25)$ & 4 & $0-1$ \\
\hline & A. obstetricans & $20,54(6,17)$ & 22 & $0-107$ \\
\hline & D. galganoi & $1,37(0,53)$ & 8 & $0-4$ \\
\hline & P. punctatus & $1,75(1,03)$ & 4 & $0-4$ \\
\hline & B. spinosus & $2,60(1,07)$ & 5 & $0-6$ \\
\hline & E. calamita & $20,00(16,71)$ & 7 & $0-120$ \\
\hline & H. molleri & $15,12(12,82)$ & 16 & $0-207$ \\
\hline & P. perezi & $6,71(3,34)$ & 7 & $0-24$ \\
\hline \multirow{9}{*}{$\begin{array}{l}\text { Adultos } \\
\text { (década } \\
2010 \text { ) }\end{array}$} & L. helveticus & $36,40(10,16)$ & 30 & $0-235$ \\
\hline & T. marmoratus & $5,83(1,39)$ & 30 & $0-33$ \\
\hline & I. alpestris & $3,33(1,96)$ & 6 & $0-13$ \\
\hline & A. obstetricans & $0,38(0,21)$ & 13 & $0-2$ \\
\hline & D. galganoi & 0 & 1 & \\
\hline & B. spinosus & $0,14(0,09)$ & 14 & $0-1$ \\
\hline & E. calamita & 0 & 4 & \\
\hline & H. molleri & $4,66(2,83)$ & 15 & $0-38$ \\
\hline & P. perezi & $9,63(5,57)$ & 22 & $0-113$ \\
\hline \multirow{11}{*}{$\begin{array}{l}\text { Larvas } \\
\text { (década } \\
2010 \text { ) }\end{array}$} & S. salamandra & $3,14(0,93)$ & 21 & $0-14$ \\
\hline & L. helveticus & $16,56(6,50)$ & 25 & $0-145$ \\
\hline & T. marmoratus & $24,25(9,19)$ & 24 & $0-178$ \\
\hline & I. alpestris & $0,20(0,20)$ & 5 & $0-1$ \\
\hline & A. obstetricans & $19,64(6,74)$ & 25 & $0-140$ \\
\hline & D. galganoi & 0 & 7 & \\
\hline & P. punctatus & 0 & 6 & \\
\hline & B. spinosus & $0,40(0,40)$ & 5 & $0-2$ \\
\hline & E. calamita & 0 & 6 & \\
\hline & H. molleri & $31,19,(15,35)$ & 21 & $0-293$ \\
\hline & P. perezi & $4,44(1,84)$ & 9 & $0-14$ \\
\hline
\end{tabular}


ticus, que creció entre 1995 y $1999(t=-3,076 ; P=0,005)$ y las de $A$. obstetricans $(U=8,5 ; P=0,028)$ y $B$. spinosus $(U=2,5 ; P=0,011)$, que decrecieron en 1999.

Agrupados los datos para comparar su evolución entre décadas ( $n=15$ humedales muestreados cada año), se comprobó que las únicas variaciones producidas en las poblaciones de adultos afectaron a $T$. marmoratus $(U=201$; $P=0,004)$, que creció, y a $B$. spinosus $(U=49 ; P=0,029)$ y $P$. perezi $(U=85 ; P=0,001)$, que decrecieron. Entre las larvarias, se estimaron crecimientos significativos en las poblaciones de S. salamandra $(U=68,5 ; P<0,000)$, L. helveticus $(U=169,5 ; P=0,030)$ y $T$. marmoratus $(U=149 ; P=0,009)$ (Tabla 2$)$. En los humedales investigados, ciertas especies como D. galganoi, Pelodytes punctatus (Daudin, 1802) y E. calamita sólo se detectaron en la década de 1990, si bien se observaron larvas de esta última especie en 2010, en un humedal que no entró a formar parte del censo.

\section{Censo poblacional en Izki}

En la década de 2010 las diferencias en las abundancias máximas medias también fueron en Izki altamente significativas, tanto en las poblaciones adultas $(H=34,57$; $P<0,000)$ como en las larvarias $(H=27,75 ; P<0,000)$. Los valores más altos en poblaciones adultas los presentaron $L$. helveticus y $P$. perezi, mientras que los valores más altos de larvas fueron para $H$. molleri, $R$. dalmatina y $A$. obstetricans (Tabla 3). La única especie cuya estimación de la población adulta creció en la década de 2010 fue $A$. obstetricans ( $U=3 ; P=0,023)$, mientras que las larvarias de $L$. helveticus $(\mathrm{H}=5,67 ; \mathrm{P}=0,046)$ y $H$. molleri $(\mathrm{t}=3,12 ; \mathrm{P}=0,008)$ decrecieron (Tabla 3). Las estimaciones de las poblaciones adultas de $T$. marmoratus, $A$. obstetricans, $B$. spinosus y H. molleri decrecieron de la década de 1990 a la de 2010 (Mann-Whitney: $P<0,040$; $\mathrm{n}=6$ humedales cada año). Sólo se detectaron poblaciones de adultos de $P$. punctatus, D. galganoi y E. calamita en la década de 1990, época en la que no se realizaron censos de larvas. En la de 2010, la presencia en los censos larvarios de estas dos últimas especies fue testimonial, y P. punctatus desapareció completamente de los muestreos.

Tabla 2.- Estadística descriptiva de la abundancia máxima de las poblaciones de adultos y larvarias en el Parque Natural de Valderejo en las décadas de 1990 y 2010, estimada como densidad relativa ( ${ }^{\circ}$ individuos / 20 minutos de muestreo) y calculada para los humedales comunes a ambas.

Table 2.- Descriptive statistics of the maximum abundance of adult and larval populations in the Natural Park of Valderejo in the decades of 1990 and 2010, estimated as relative density (no. individuals / 20 minutes of sampling) and calculated for the wetlands common to both. 


\begin{tabular}{|c|c|c|c|c|}
\hline Estadio & Especie & $\begin{array}{c}\text { Media } \\
\text { (error estándar) }\end{array}$ & $n$ & Rango \\
\hline \multirow{11}{*}{$\begin{array}{l}\text { Adultos } \\
\text { (década } \\
\text { 1990) }\end{array}$} & L. helveticus & $18,00(3,79)$ & 6 & $12-36$ \\
\hline & T. marmoratus & $7,66(4,93)$ & 6 & $0-32$ \\
\hline & A. obstetricans & $8,33(3,44)$ & 6 & $0-24$ \\
\hline & D. galganoi & $8,00(5,16)$ & 6 & $0-32$ \\
\hline & P. punctatus & $2,33(1,66)$ & 6 & $0-10$ \\
\hline & B. spinosus & $9,66(3,44)$ & 6 & $0-20$ \\
\hline & E. calamita & $1,33(0,84)$ & 6 & $0-4$ \\
\hline & H. molleri & $100,80(46,88)$ & 5 & $0-250$ \\
\hline & R. temporaria & $105,66(98,92)$ & 6 & $0-600$ \\
\hline & R. dalmatina & $37,66(15,58)$ & 6 & $0-86$ \\
\hline & P. perezi & $31,66(16,56)$ & 6 & $0-112$ \\
\hline \multirow{11}{*}{$\begin{array}{l}\text { Adultos } \\
\text { (década } \\
2010 \text { ) }\end{array}$} & L. helveticus & $20,07(6,08)$ & 13 & $0-68$ \\
\hline & T.marmoratus & $0,69(0,34)$ & 13 & $0-4$ \\
\hline & A.obstetricans & $0,30(0,15)$ & 10 & $0-1$ \\
\hline & D. galganoi & 0 & 18 & \\
\hline & P. punctatus & 0 & 18 & \\
\hline & B. spinosus & $1,05(0,74)$ & 18 & $0-13$ \\
\hline & E. calamita & 0 & 18 & \\
\hline & H. molleri & $0,46(0,29)$ & 15 & $0-4$ \\
\hline & R. temporaria & $1,44(1,22)$ & 18 & $0-22$ \\
\hline & R. dalmatina & $0,05(0,05)$ & 18 & $0-1$ \\
\hline & P.perezi & $9,27(3,74)$ & 18 & $0-65$ \\
\hline \multirow{10}{*}{$\begin{array}{l}\text { Larvas } \\
\text { (década } \\
2010 \text { ) }\end{array}$} & L. helveticus & $15,53(5,98)$ & 28 & $0-156$ \\
\hline & T. marmoratus & $3,73(1,26)$ & 19 & $0-18$ \\
\hline & A. obstetricans & $27,53(13,21)$ & 15 & $1-184$ \\
\hline & D. galganoi & $0,33(0,21)$ & 6 & $0-1$ \\
\hline & B. spinosus & $1,66(0,91)$ & 6 & $0-5$ \\
\hline & E. calamita & $3,33(3,33)$ & 3 & $0-1$ \\
\hline & H. molleri & $134,44(112,17)$ & 9 & $0-1027$ \\
\hline & R. temporaria & $22,14(7,86)$ & 7 & $1-50$ \\
\hline & R.dalmatina & $76,40(37,37)$ & 10 & $1-391$ \\
\hline & P. perezi & $9,50(6,86)$ & 6 & $0-43$ \\
\hline
\end{tabular}

Tabla 3.- Estadística descriptiva de la abundancia máxima de las poblaciones de adultos y larvarias en el Parque Natural de Izki en las décadas de 1990 y 2010, estimada como densidad relativa ( $n^{\circ}$ individuos / 20 minutos de muestreo) y calculada para los humedales comunes a ambas. En la década de 1990 no se capturaron larvas en Izki. En las estimaciones larvarias de la década de 2010 se amplió la muestra de humedales hasta en seis unidades.

Table 3.- Descriptive statistics of the maximum abundance of adult and larval populations in the Natural Park of Izki in the decades of 1990 and 2010, estimated as relative density (No. individuals / 20 minutes of sampling) and calculated for the wetlands common to both. In the 1990s, no larvae were captured in Izki. In the larval estimates for the 2010s, the sample of wetlands was expanded by up to six units. 


\section{Discusión}

El inventario de especies de anfibios en las charcas comparadas en los dos parques habría descendido en una sola especie en cada uno de ellos, desde la década de 1990. En ninguna de esas charcas fue detectada la presencia de $S$. salamandra en esa época, si bien ésta se encontraba entonces presente en otras charcas de los parques. El periodo de ocho años comprendido entre los dos extremos del seguimiento realizado en la década de 2010 no es suficiente para extraer conclusiones definitivas sobre la tendencia de las poblaciones de anfibios en los dos parques alaveses, pero aportaría una información valiosa para detectar procesos que pueden determinar el futuro de las especies y sobre la conservación de una parte importante de los humedales que acogen su reproducción. Sin embargo, el análisis comparativo, según la misma metodología, con los censos realizados en la década de 1990 (Onrubia et al., 1996; CRN, 1999; Onrubia et al., 2007) ampliaría la perspectiva a 18 años en Izki y 22 en Valderejo, aunque deban matizarse aspectos referentes a la efectividad interpretativa de los resultados en especies que, por sus rasgos ecológicos y de conducta, no coincidieron plenamente, en alguna de sus fases vitales, con la época en que se realizaron los censos. Dichos resultados deben ser tomados, por tanto, con cautela, en el caso de las poblaciones adultas de $A$. obstetricans, $B$. spinosus o $H$. molleri en Izki (que habrían decrecido), o directamente no ser tenidos en cuenta en el de las de $R$. dalmatina y $R$. temporaria en dicho parque. De haberse producido variaciones en su estatus, éste podría ser explicado por las fluctuaciones naturales de sus poblaciones y la climatología particular coincidente con ciertos años de muestreo, aunque estas apreciaciones deberán ir siendo perfiladas en futuras campañas periódicas de seguimiento. Por el contrario, la desaparición de los humedales donde fueron registradas en la década de 1990 de dos especies caracterizadoras del medio mediterráneo, como D. galganoi y $P$. punctatus, sí estaría respondiendo a una situación de declive, que ya es de por sí muy grave y generalizada para las poblaciones de $D$. galganoi en la cuenca Alta del Ebro (Garin-Barrio et al., 2013), pero más circunstancial para P. punctatus, que fue localizada en la década de 1990 en cuatro humedales de la zona cultivada de Valderejo. Aun cuando posteriormente estos humedales no se hayan muestreado o los censos se hayan hecho fuera de la época de actividad, la especie tampoco se ha llegado a detectar por escuchas. Ambas especies se encuentran localizadas en Valderejo e Izki en uno de sus bordes septentrionales de distribución (SIARE, 2018), en un ambiente montano que no es su preferido y sustentadas en poblaciones de muy baja densidad. Puesto que el nivel de protección en los parques naturales es razonablemente bueno, posiblemente los causantes de este descenso puedan tener que ver con fenómenos relacionados con el cambio climático, y en algún caso (especialmente en Valderejo) por la existencia en el interior de los parques de zonas ocupadas por la ganadería extensiva (pastizales que recibirían aportes de fertilizantes y agroquímicos). Discoglossus galganoi habría desaparecido de Valderejo, cuando en los muestreos de 1995- 
1999 estaba presente en, al menos, ocho humedales repartidos en ambiente forestal, rasos de montaña y zona cultivada (pastizales ganaderos) (Onrubia et al., 1996; CRN, 1999). En Izki aparecía en el año 2000 en siete humedales, y ya en 2010 sólo pudo ser observado, de forma testimonial (alguna larva), en dos humedales. Por su parte, E. calamita —especie también representativa del medio mediterráneo- estuvo presente hasta en 15 humedales de Valderejo en 1995 (Onrubia et al., 1996), ubicados también en todos los ambientes del parque, habiéndose censado más recientemente en tan sólo un humedal. Probablemente, las condiciones climatológicas de 2017, que impidieron la recarga de la única masa de agua en la que estaba siendo observado, hayan impedido su detección. Sin embargo, la especie sigue estando presente en el parque, en enclaves sólo censados en la década de 1990 que no han sido considerados en los muestreos de la década de 2010 (C. Tejado, Instituto Alavés de la Naturaleza, com. pers.).

El aumento de los registros de I. alpestris en Valderejo, con respecto a los de la década de 1990 — una especie cuya abundancia ha sido recientemente revisada (Gosá et al., 2017b) incluyendo las poblaciones de este parque_-, podría responder tanto a una expansión real de sus núcleos reproductores como a una coincidencia de los muestreos en charcas especialmente favorecidas por unas condiciones climáticas favorables en 2017. La tendencia de la mayor parte de las restantes especies en el parque parece estar estabilizada, y sólo se apreciaría crecimiento en la de T. marmoratus (refrendada en su población larvaria) y decrecimiento en las de $B$. spinosus y $P$. perezi, si bien la interpretación de la evolución de estas poblaciones estaría sujeta a los comentarios ya expuestos más arriba.

\section{Agradecimientos}

Jonathan Rubines (Diputación Foral de Álava) puso a nuestra disposición información útil para la elaboración del estudio. Las respectivas guarderías de los parques facilitaron nuestros desplazamientos en ellos. Los muestreos se realizaron bajo los preceptivos permisos del Servicio de Patrimonio Natural de la Diputación Foral de Álava, entidad financiadora del estudio.

\section{Bibliografía citada}

Araújo, M.B., Alagador, D., Cabeza, M., Nogués-Bravo, D., Thuiller, W. 2011. Climate change threatens European conservation areas. Ecol. Letters 14: 484-492.

Crespo Diaz, A. 2008. Identificación de poblaciones, distribución y estado de conservación 
del sapo de espuelas (Pelobates cultripes) y los sapillos pintojos (Discoglossus spp.) en Álava. Diputación Foral de Álava. Vitoria-Gasteiz. Inédito.

Crespo Diaz, A., González Ochoa, S., Iraola, A. 2007. Identificación de poblaciones, distribución y estado de conservación de los sapillos pintojos (Discoglossus sp.) en el País Vasco. Gobierno Vasco. Vitoria-Gasteiz. Inédito.

Crespo Diaz, A., Tamayo, I. 2007. Áreas importantes para los Anfibios y Reptiles en el País Vasco. Gobierno Vasco. Vitoria-Gasteiz. Inédito.

CRN. 1999. Programa de seguimiento de las comunidades faunísticas del Parque Natural de Valderejo, Álava. Peces y anfibios. Gobierno Vasco. Vitoria-Gasteiz. Inédito.

Ekos. 2006. Revisión del estado de conservación de las poblaciones de anfibios de los humedales de Salburua. CEA, Ayuntamiento de Vitoria-Gasteiz.

Etxezarreta, J., Martínez-Jorquera, J., Txintxurreta, A., Rubio, X. 2007. Seguimiento de la ranita meridional, Hyla meridionalis (Boettger, 1874) del País Vasco en el periodo 1998-2006. Munibe Supl. 25: 238-248.

Garin-Barrio, I., San Sebastián, O., Océn-Ratón, M., Rubio, X. 2007. Estado de conservación de las poblaciones de sapo corredor (Bufo calamita) en la costa vasca. Munibe Supl. 25: 292301.

Garin-Barrio, I., Zaldívar, C., Zaldívar, R., Zuazo, Ó., Álvarez, J., Gosá, A., Crespo-Diaz, A., Tejado, C., Potes, M.E. 2013. El declive del sapillo pintojo meridional en el Alto Ebro. Quercus 332: $26-33$.

Gosá, A. 2011. Situación del sapillo pintojo meridional en los Parques Naturales de Izki y Valderejo. Campaña 2011. Diputación Foral de Álava. Vitoria-Gasteiz. Inédito.

Gosá, A., Garin-Barrio, I. 2017. Seguimiento de anfibios en el Parque Natural de Izki. Campaña 2017. Diputación Foral de Álava. Vitoria-Gasteiz. Inédito.

Gosá, A., Garin-Barrio, I., Cabido, C., Laza-Martínez, A. 2017a. Seguimiento de anfibios en el Parque Natural de Valderejo. Campaña 2017. Diputación Foral de Álava. Vitoria-Gasteiz. Inédito.

Gosá, A., Garin-Barrio, I., Laza-Martínez, A. 2017b. Distribución y situación actual de la población oriental ibérica de Ichthyosaura alpestris. Bol. Asoc. Herpetol. Esp. 28(2): 57-65.

Gosá, A., Iraola, A. 2010a. Estudio preliminar de los efectos del ganado extensivo sobre los anfibios en el Parque Natural de Izki. 1. Selección de charcas y primeros datos poblacionales. Diputación Foral de Álava. Vitoria-Gasteiz. Inédito.

Gosá, A., Iraola, A. 2010b. Determinación de las características poblacionales y estado de conservación del tritón alpino y el sapillo pintojo en el Parque Natural de Valderejo. Diputación Foral de Álava. Vitoria-Gasteiz. Inédito.

Heyer, W.R., Donnelly, M.A., McDiarmid, R.W., Hayek, L.A., Foster, M.S. 1994. Measuring and monitoring biological diversity. Standard methods for Amphibians. Smithsonian Institution Press, Washington and London. 
Mateo, J.A. 2002. Áreas importantes para la herpetofauna española. En: Atlas y Libro Rojo de los Anfibios y Reptiles de España. Pleguezuelos, J.M., Márquez, R., Lizana, M. (eds.): 483500. Dirección General de Conservación de la Naturaleza-Asociación Herpetológica Española ( $2^{a}$ impresión). Madrid.

Olano, I., Ruiz de Azua, N., Fernández, J.M., Arrayago, M.J., Bea, A. 2007. Estado de conservación de poblaciones periféricas de anfibios: tritón pirenaico Euproctus asper y sapo de espuelas Pelobates cultripes en la Comunidad Autónoma del País Vasco. Munibe Supl. 25: 66-73.

Onrubia, A., Sáenz de Buruaga, M, Campos, M.A., Lucio, A.J., Purroy, F.J., Balmorí, A., Fernández, J. 1996. Estudio faunístico del Parque Natural de Valderejo (Álava). Informe técnico $n^{\circ}$ 71. Gobierno Vasco. Vitoria-Gasteiz.

Onrubia, A., Sáenz de Buruaga, M, Campos, M.A., Balmorí, A. 2007. Herpetofauna del Parque Natural de Izki (Álava, País Vasco). Munibe Supl. 25: 58-64.

Pagoa. 2010. Seguimiento de las poblaciones de anfibios del humedal de Salburua (Álava). Año 2009. CEA, Ayuntamiento de Vitoria-Gasteiz.

Santini, L., Saura, S., Rondinini, C. 2015. Connectivity of the global network of protected areas. Diversity and Distributions. https://doi.org/10.1111/ddi.12390.

Santos, X., Carretero, M.Á., Llorente, G.A., Montori, A. 1998. Inventario de las áreas importantes para los anfibios y reptiles de España. Colección Técnica. ICONA. Madrid.

SIARE (Servidor de Información de Anfibios y Reptiles de España). 2018. http://siare.herpetologica.es/bdh [Consulta: 22 enero 2018].

Fecha de recepcion/Date of reception: 12/03/2018

Fecha de aceptacion/Date of acceptance: 30/11/2018

Editor Asociado/Associate editor: Ivan de la Hera 